\title{
COMPORTAMENTO GERMINATIVO DE SEMENTES DE Talisia subalbens (Mart.) Radlk. (Sapindaceae) SUBMETIDAS A DIFERENTES TEMPERATURAS E CONDIÇÕES DE SECAGEM
}

\author{
Behaviour of germination of Talisia subalbens (Mart.) Radlk. (Sapindaceae) seeds \\ submitted to different temperatures and drying conditions
}

\author{
Hilton Morbeck Oliveira ${ }^{1}$, Fernanda Carlota Nery ${ }^{2}$, Amauri Alves de Alvarenga ${ }^{3}$, \\ João Paulo Delfino Barbosa ${ }^{4}$, Daniel Diego Costa Carvalho ${ }^{4}$
}

\begin{abstract}
RESUMO
A Talisia subalbens (Mart.) Radlk. (Sapindaceae) é uma espécie arbustiva apontada como endêmica do Mato Grosso. Objetivou-se neste estudo avaliar os efeitos da temperatura e condições de secagem sobre a germinação de sementes de T. subalbens. No primeiro experimento avaliou-se a germinação das sementes em diferentes regimes térmicos: $15^{\circ} \mathrm{C}-25^{\circ} \mathrm{C}$ e $25^{\circ} \mathrm{C}-35^{\circ} \mathrm{C}$ (alternadas), $25^{\circ} \mathrm{C}$ e $35^{\circ} \mathrm{C}$ (constante), e no segundo experimento, as sementes foram submetidas à diferentes condições de secagem: rápida (estufa à $35^{\circ} \mathrm{C}$ ) e lenta (temperatura ambiente à $28 \pm 2^{\circ} \mathrm{C}$ ). Os testes de germinação foram realizados em câmaras do tipo B.O.D., sob fotoperíodo de 12 horas, utilizando-se vermiculita como substrato. O delineamento foi o inteiramente casualizado, com quatro repetições de 25 sementes cada amostra. Os valores médios de porcentagem de germinação e índice de velocidade de emergência (IVE) foram comparados pelo teste de Tukey $(5 \%)$. As sementes germinadas a $25^{\circ} \mathrm{C}, 35^{\circ} \mathrm{C}$ e $25^{\circ} \mathrm{C}-35^{\circ} \mathrm{C}$ apresentaram maiores porcentagens de germinação, não diferindo entre si, contudo, maior vigor foi observado à $35^{\circ} \mathrm{C}$, sendo esta considerada a temperatura ótima de germinação da espécie. Os menores valores de germinação e IVE ocorreram a $15^{\circ} \mathrm{C}-25^{\circ} \mathrm{C}$. O grau de umidade inicial das sementes foi de $48 \%$. A velocidade de secagem das sementes afeta negativamente a porcentagem de germinação, sendo o teor crítico de grau de umidade de $25 \%$, e a secagem rápida mais favorável para a espécie.
\end{abstract}

Termos para a indexação: Cerrado, secagem, emergência, Talisia subalbens.

\begin{abstract}
Talisia subalbens (Mart.) Radlk. (Sapindaceae), a shrubby species, is considered endemic to Mato Grosso. The aim of this study was to evaluate the responses of its seeds to factors such as the optimum germination temperature and the effect of different drying conditions on the physiological quality of the seeds. In the first experiment, seed germination under different temperatures was evaluated: $15^{\circ} \mathrm{C}-25^{\circ} \mathrm{C}$ and $25^{\circ} \mathrm{C}-35^{\circ} \mathrm{C}$ (alternate), $25^{\circ} \mathrm{C}$ and $35^{\circ} \mathrm{C}$ (constant). In the second experiment, the seeds were submitted to different drying conditions: fast (oven at $35^{\circ} \mathrm{C}$ ) and slow (room temperature at $28 \pm 2^{\circ} \mathrm{C}$ ). The germination tests were performed in a B.O.D-type chamber, under a photoperiod of 12 hours, using vermiculite as the substrate. The experimental design was the completely randomized with four replications of 25 seeds for each sample, and the average values of germination at the different temperatures compared by the Tukey test $(5 \%)$. Seeds germinated at $25^{\circ} \mathrm{C}, 35^{\circ} \mathrm{C}$, and $25^{\circ} \mathrm{C}-35^{\circ} \mathrm{C}$ presented higher percentages of germination, not differing from each other. However, greater vigor was found at $35^{\circ} \mathrm{C}$, this being considered the optimum temperature. The lowest values of germination and germination index (GI) occurred at $15^{\circ} \mathrm{C}-25^{\circ} \mathrm{C}$. The initial moisture of the seeds was $48 \%$. The drying of the seeds negatively affected the germination, with the critic moisture content of $25 \%$ and the fast drying being more favorable to the germination.
\end{abstract}

Index terms: Cerrado, drying, emergency, Talisia subalbens.

(Recebido em 10 de agosto de 2007 e aprovado em 14 de fevereiro de 2008)

\section{INTRODUÇÃO}

Talisia subalbens (Mart.) Radlk (Sapindaceae), conhecida como cascudo ou cascudinho é uma espécie arbustiva apontada como endêmica do estado do Mato Grosso, restrita ao cerrado da Chapada dos Guimarães
(MT), a qual tem sido foco da ação predatória do homem e, portanto, seriamente ameaçada de extinção. Diante deste fato, torna-se relevante o desenvolvimento de ações no âmbito da preservação de recursos naturais e, especialmente, no campo da propagação, visando à perpetuação desta espécie no seu hábitat.

'Biólogo, Professor Adjunto - Departamento de Biologia - Universidade Federal do Mato Grosso/UFMT - Campus UFMT - 78000-000 - Rondonópolis, MT sahbeck@globo.com

${ }^{2}$ Doutora em Fisiologia Vegetal - Departamento de Biologia/DBI - Universidade Federal de Lavras/UFLA - Cx. P. 3037 - $37200-000$ - Lavras, MG fernandacarlota@yahoo.com.br

${ }^{3}$ Engenheiro Agrônomo, Professor Titular - Departamento de Biologia/DBI - Universidade Federal de Lavras/UFLA - Cx. P. 3037 - $37200-000$ - Lavras, MG amauriaa@ufla.br

${ }^{4}$ Engenheiros Agrônomos - Departamento de Biologia/DBI - Universidade Federal de Lavras/UFLA - Cx. P. 3037 - $37200-000$ - Lavras, MG delfinojp@gmail.com; ufla-ddcc@bol.com.br 
No processo germinativo de uma semente alguns fatores, a exemplo da temperatura, devem ser considerados como um dos principais responsáveis tanto pela velocidade como pelo percentual de germinação, por afetar especialmente a velocidade de absorção de água e reativar as reações metabólicas fundamentais aos processos de mobilização de reservas e ao crescimento da plântula (BEWLEY \& BLACK, 1994). Sementes de muitas espécies requerem flutuação diária de temperatura para germinar adequadamente, a alternância de temperatura pode acelerar a germinação em sementes não dormentes (FERREIRA \& BORGHETTI, 2004; DOUSSEAU et al., 2008; NERY et al., 2007).

O teor de água é um fator determinante do comportamento das sementes recalcitrantes durante o armazenamento. Nessas sementes, a água subcelular está fortemente associada às superfícies macromoleculares, assegurando, em parte, a estabilidade de membranas e macromoléculas. A perda de água estrutural durante o processo de secagem causaria alteração de sistemas metabólicos e de membranas, resultando no início do processo de deterioração (FARRANT et al., 1997).

O conhecimento da capacidade que uma espécie tem em tolerar a perda de água é de grande importância nos processos de secagem e armazenamento. A secagem deve ser de modo que não promova uma retirada brusca de água do interior da semente, provocando desorganização e descompartimentalização cada vez maiores de todo o sistema de membranas da semente. Essa desorganização continua durante $\mathrm{o}$ armazenamento, mas, será retardada se as condições forem ideais (ROBERTS, 1973).

As sementes recalcitrantes têm sua viabilidade reduzida quando o teor de água atinge valores inferiores àqueles considerados críticos; quando iguais ou inferiores àqueles considerados letais, há perda total de viabilidade (HONG \& ELLIS, 1990). O conhecimento dos teores crítico e letal de água de uma espécie é indispensável para o planejamento e a execução da secagem e do armazenamento das sementes.

Neste contexto, objetivou-se com este estudo avaliar o comportamento germinativo de sementes de $T$. subalbens (Mart.) Radlk. submetidas a diferentes temperaturas e condições de secagem.

\section{MATERIALE MÉTODOS}

Os experimentos foram conduzidos no Laboratório de Crescimento e Desenvolvimento de Plantas, Setor de Fisiologia Vegetal, no Departamento de Biologia, na Universidade Federal de Lavras. Foram utilizadas sementes de T. subalbens (Mart.) Radlk., provenientes de frutos colhidos no período da dispersão, nos meses de novembro e dezembro de 2004, de plantas localizadas no município de Chapada dos Guimarães, MT. Sob condições de laboratório os frutos foram despolpados e, em seguida, as sementes tiveram seu tegumento removido. $\mathrm{O}$ teor de água das sementes foi determinado em estufa à $105^{\circ} \mathrm{C} \pm 3^{\circ} \mathrm{C}$, por um período de 24 horas, utilizando-se quatro repetições de 10 sementes (BRASIL, 1992). Os resultados, expressos em porcentagem, foram calculados com base no peso úmido.

Para o estudo da influência da temperatura na germinação, foram utilizados lotes de sementes com umidade inicial de $48 \%$, sendo essas submetidas a diferentes regimes térmicos de $25^{\circ} \mathrm{C}$ e $35^{\circ} \mathrm{C}$ (constantes), $15^{\circ} \mathrm{C}-25^{\circ} \mathrm{C}$ e $25^{\circ} \mathrm{C}-35^{\circ} \mathrm{C}$ (alternadas), com fotoperíodo de 12 horas. Foram feitas quatro repetições de 25 sementes cada e avaliadas diariamente quanto à porcentagem de germinação e índice de velocidade de emergência das plântulas (IVE). O teste de germinação foi realizado seguindo metodologia das Regras para Análises de Sementes (BRASIL, 1992). As sementes foram acondicionadas em caixas tipo gerbox, contendo substrato vermiculita, mantidas em câmaras de germinação modelo B.O.D. 347 Fanem ou Eletrolab. As avaliações de porcentagem de germinação foram realizadas diariamente, utilizando-se, como parâmetro de germinação, a protrusão da raiz primária com aproximadamente $5 \mathrm{~mm}$ de comprimento. O IVE foi avaliado juntamente com o experimento de germinação, sendo considerada como emersas as plântulas que apresentavam aproximadamente $3 \mathrm{~mm}$ de comprimento de parte aérea.

Para o estudo do efeito das condições de secagem sobre a germinação, as sementes com umidade inicial de $48 \%$ foram submetidas à duas condições de secagem: uma rápida, em estufa com circulação de ar à $35^{\circ} \mathrm{C}$, e lenta, sob ambiente de laboratório, a temperatura de $28 \pm 2^{\circ} \mathrm{C}$, ambas por um período de 28 dias. Amostras de 25 sementes foram tomados para a avaliação da qualidade fisiológica, quanto à porcentagem de germinação e IVE, sendo utilizada para a realização dos testes a temperatura ótima de germinação definida anteriormente (BRASIL, 1992).

Os experimentos foram conduzidos em delineamento inteiramente casualizado (DIC). Os dados foram previamente submetidos aos testes de normalidade dos resíduos e homocedasticidade das variâncias. Os dados de germinação foram transformados em arco seno $(\mathrm{x} / 100)^{0,5}$ e o índice de velocidade de emergência (IVE) calculado segundo Maguire (1962) e os dados transformados em $(x+0,5)^{0,5}$ (ZAR, 1999). Os dados foram submetidos à análise de variância e as médias comparadas pelo teste de Tukey, a 5\% de probabilidade e para o 
experimento de secagem as médias foram comparadas pelo teste de Scott \& Knott (1974) $(\mathrm{p}<0,05)$, mediante o uso do programa estatístico SISVAR (FERREIRA, 2000).

\section{RESULTADOS E DISCUSSÃO}

Independente da temperatura as sementes de $T$. subalbens apresentaram porcentagem máxima de germinação a $35^{\circ} \mathrm{C}, 25^{\circ} \mathrm{C}$ e $25^{\circ} \mathrm{C}-35^{\circ} \mathrm{C}$, enquanto que a $15^{\circ} \mathrm{C}-25^{\circ} \mathrm{C}$, os valores de germinação atingiram a ordem dos $95 \%$ (Figura 1).

Quanto ao IVE, sementes submetidas à $35^{\circ} \mathrm{C}$ obtiveram germinação mais rápida, seguida das sementes à $25^{\circ} \mathrm{C}$ e $25^{\circ} \mathrm{C}-35^{\circ} \mathrm{C}$, sendo essas não diferentes entre si estatisticamente. Todavia, o menor valor de IVE foi observado a $15^{\circ} \mathrm{C}-25^{\circ} \mathrm{C}$, em que as sementes apresentaram um atraso considerável na germinação (Figura 2).

A menor porcentagem de germinação e IVE encontrados para sementes submetidas à temperatura alternada de $15^{\circ} \mathrm{C}-25^{\circ} \mathrm{C}$, possivelmente, ocorreu em razão da exposição das sementes à temperatura de $15^{\circ} \mathrm{C}$, consideravelmente baixa, na qual diversos autores relatam reduzir a porcentagem de germinação, bem como, retardar o processo, em razão da redução das atividades enzimáticas envolvidas no metabolismo da semente (BEWLEY \& BLACK, 1994). Segundo Scatena et al. (1996), a correlação positiva entre porcentagem e velocidade de emergência

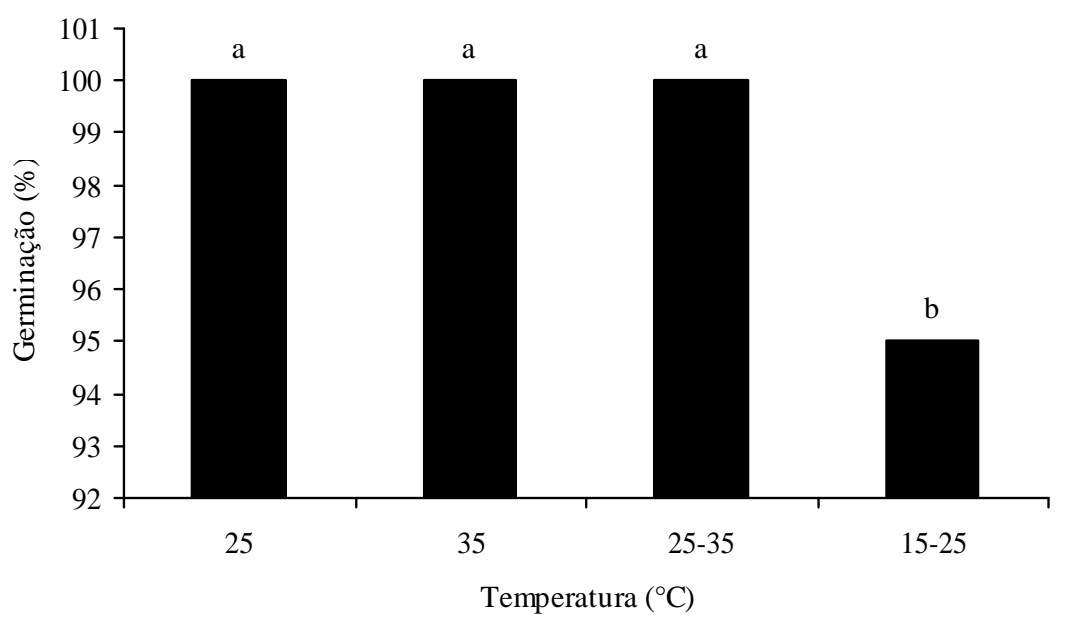

Figura 1 - Germinação (\%) de sementes T. subalbens submetidas a diferentes regimes térmicos. Médias com a mesma letra não diferem entre si pelo teste de Tukey $(5 \%)$.

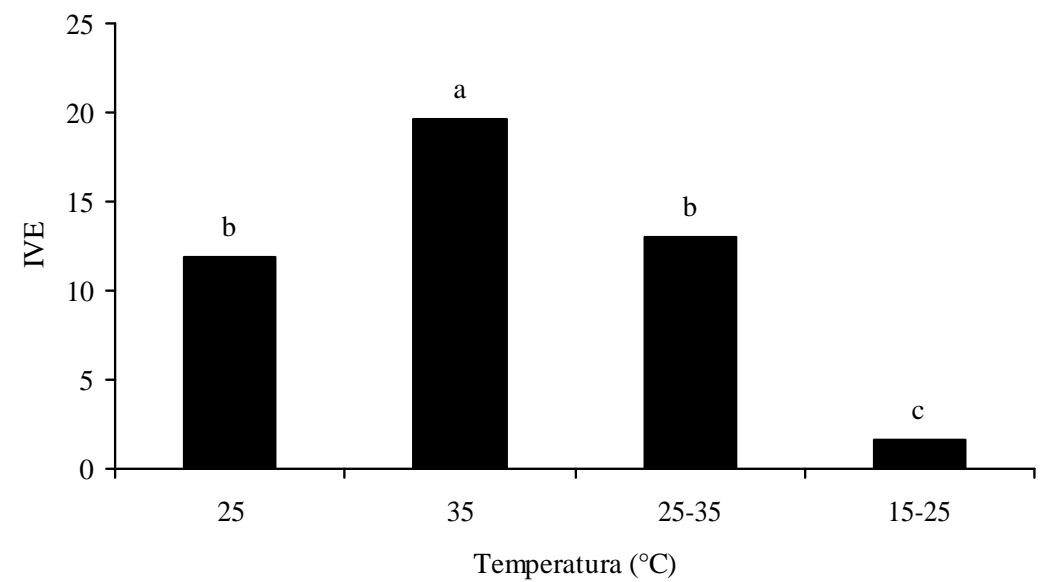

Figura 2 - Índice de velocidade de emergência (IVE) de sementes de T. subalbens submetidas aos diferentes regimes térmicos. Médias seguidas pela mesma letra não diferem entre si pelo teste de Tukey (5\%). 
das sementes garante o sucesso na obtenção e no estabelecimento das plântulas. Por outro lado, sob temperaturas altas, a velocidade de absorção de água bem como as atividades enzimáticas, tornam-se mais elevadas, fazendo com que as sementes germinem mais rapidamente (CARVALHO \& NAKAGAWA, 2000). Para as sementes de $T$. subalbens a máxima germinação ocorreu entre $25^{\circ} \mathrm{C}$ e $35^{\circ} \mathrm{C}$ e a maior velocidade de emergência a $35^{\circ} \mathrm{C}$.

De acordo com a literatura, sementes de diferentes espécies florestais e classificação ecológica apresentam respostas diferenciais à temperatura do meio de germinação. Lima Júnior (2004), trabalhando com Cupania vernalis Camb., verificou redução no índice de velocidade de germinação quando as sementes foram submetidas à $15^{\circ} \mathrm{C}-25^{\circ} \mathrm{C}$. Araújo Neto et al. (2003), trabalhando com Acacia polyphylla DC., observaram que as sementes apresentaram maior percentagem final de germinação a $25^{\circ} \mathrm{C}$, tendo, entretanto, inibido a germinação sob temperaturas superiores a $35^{\circ} \mathrm{C}$ e inferiores a $25^{\circ} \mathrm{C}$. Em duas espécies de Albizia, temperaturas de $20^{\circ} \mathrm{C}$ e $25^{\circ} \mathrm{C}$ proporcionaram maiores porcentagens e velocidades de germinação (TIGABU \& ODEM, 2001). Segundo Bewley \& Black (1994), sementes de diferentes espécies apresentam grande variação no comportamento quanto à temperatura de germinação, apresentando uma faixa ótima situada entre as temperaturas da época propícia à germinação no seu hábitat (VILLALOBOS \& PELÀEZ, 2001). A variabilidade de respostas quanto ao requerimento de temperatura é um reflexo da adaptação das espécies ao ambiente de ocorrência (THOMPSON, 1970). Essa resposta é de origem genética, decorrente, principalmente, da plasticidade fenotípica, que permite a germinação das sementes e o estabelecimento das plântulas sob diversas condições ambientais (MALUF \& MARTINS, 1991).

O processo de secagem das sementes proporcionou decréscimo linear na porcentagem total de germinação, independente do ambiente de secagem (Tabela 1).

Pelos dados da Tabela 1, observa-se que em condições de secagem rápida (estufa à $35^{\circ} \mathrm{C}$ ), no $4^{\circ}$ e $8^{\circ}$ dia de secagem, os teores de água foram respectivamente, de $45 \%$ e $40 \%$. A porcentagem total de germinação não diferiu da testemunha no tempo zero, quando as sementes apresentavam $48 \%$ de teor de água inicial. A partir do $12^{\circ}$ dia de secagem rápida, em estufa à $35^{\circ} \mathrm{C}$, a porcentagem total de germinação decresceu em todas as avaliações. Entretanto, durante a secagem lenta, em ambiente de laboratório a $28^{\circ} \mathrm{C}$, no $4^{\circ}$ dia de secagem, observou-se decréscimo de $11,7 \%$ na porcentagem total de germinação em relação à testemunha, reduzindo essa porcentagem de germinação até o $20^{\circ}$ dia de secagem. Aos 24 e 28 dias de secagem, as sementes apresentaram a menor porcentagem de germinação, não diferindo entre si. O teor crítico de umidade para a espécie em estudo foi de $25 \%$, com as sementes apresentando germinação inferior a $30 \%$. Esse tipo de comportamento é típico de sementes recalcitrantes. Resultados semelhantes foram observados por Vieira (2005), em sementes de Cupania vernalis, cuja germinação passou a ser limitada em níveis de umidade em torno de $11 \%$. Dayal \& Kaveriappa (2000) também constataram que, para diversas espécies, o limite de tolerância das sementes à dessecação no grupo das recalcitrantes é variável, como foi observado para Hopea parviflora Bedd. e Hopea ponga (Dennst.) Mabb. Segundo Dayal \& Kaveriappa (2000), são de grande importância os estudos de comportamentos das

Tabela 1 - Teor de água e porcentagem de germinação de sementes de T. subalbens, submetidas a diferentes condições e períodos de secagem.

\begin{tabular}{cccc}
\hline \multirow{2}{*}{$\begin{array}{c}\text { Período de secagem } \\
\text { (dias) }\end{array}$} & $\begin{array}{c}\text { Teor de água } \\
(\%)\end{array}$ & $\begin{array}{c}\text { Rápida } \\
\left.\text { (estufa à 35 }{ }^{\circ} \mathrm{C}\right)\end{array}$ & $\begin{array}{c}\text { Lenta } \\
\text { (ambiente à 28 }\end{array}$ \\
\hline 28 & 15 & $5,0 \mathrm{f}$ & $13,3 \mathrm{~g}$ \\
24 & 20 & $11,7 \mathrm{e}$ & $15,0 \mathrm{~g}$ \\
20 & 25 & $30,0 \mathrm{~d}$ & $23,3 \mathrm{f}$ \\
16 & 30 & $38,3 \mathrm{c}$ & $41,7 \mathrm{e}$ \\
12 & 35 & $83,3 \mathrm{~b}$ & $60,0 \mathrm{~d}$ \\
8 & 40 & $96,7 \mathrm{a}$ & $68,7 \mathrm{c}$ \\
4 & 45 & $96,7 \mathrm{a}$ & $88,3 \mathrm{~b}$ \\
0 & 48 & $100,0 \mathrm{a}$ & $100,0 \mathrm{a}$ \\
\hline
\end{tabular}

Médias seguidas pela mesma letra não diferem entre si, pelo teste de $\operatorname{Scott-Knott~}(\mathrm{p}<0,05)$. 
sementes no que diz respeito à sensibilidade a dessecação, para definir as melhores condições de armazenamento. Estudos recentes realizados por Nery (2006) em Calophyllum brasiliense Camb. mostraram que a redução no grau de umidade das sementes, de $46 \%$ para $28 \%$, não afetou significativamente o desempenho fisiológico das sementes, proporcionando variação na germinação de apenas $16 \%$. Todavia, sementes com grau de umidade abaixo de $21 \%$ tiveram acentuada redução na porcentagem de germinação. Resultados encontrados para a espécie em estudo foram semelhantes aos obtidos por Verghese et al. (2002), estudando o comportamento de sementes de Madhuca indica J.F. Gmel sob diferentes condições de secagem e armazenamento, estes autores verificaram que sementes recém-colhidas com grau de umidade de aproximadamente $53 \%$, e sementes desidratadas em condições de ambiente até $39 \%$ de grau de umidade mantiveram viabilidade máxima. Entretanto, sementes desidratadas a $37 \%$ de grau de umidade em sílica gel tiveram sua germinabilidade reduzida a $11 \%$. Segundo Kundu \& Kachari (2003) sementes de Aquilaria agollocha Roxb. submetidas a diferentes condições de desidratação apresentaram redução acentuada na porcentagem de germinação ao atingirem valores de $35 \%$ e $20 \%$ de grau de umidade. Entretanto, sementes desidratadas a $7 \%$ e $11 \%$ de grau de umidade, tiveram perda total de viabilidade. Os autores observaram ainda que a desidratação rápida favorece a sobrevivência das sementes com teores de umidade menores quando comparadas com as sementes submetidas à desidratação lenta.

\section{CONCLUSÕES}

Sementes de Talisia subalbens apresentaram germinação máxima na faixa de $25^{\circ} \mathrm{C}$ a $35^{\circ} \mathrm{C}$. As sementes dessa espécie são sensíveis à dessecação, apresentando grau crítico de umidade próximo a $25 \%$. A taxa de secagem afeta a porcentagem de germinação, sendo mais favorável a secagem rápida das sementes em estufa à $35^{\circ} \mathrm{C}$.

\section{REFERÊNCIAS BIBLIOGRÁFICAS}

ARAÚJO NETO, J. C.; AGUIAR, I. B.; FERREIRA, V. M. Efeito da temperatura e da luz na germinação de sementes de Acacia poluphylla DC. Revista Brasileira de Botânica, São Paulo, v. 26, n. 2, p. 249-256, abr./jun. 2003.

BEWLEY, J. D.; BLACK, M. Seeds: physiology of development and germination. New York: Plenum, 1994. $445 \mathrm{p}$.
BRASIL. Ministério da Agricultura e da Reforma Agrária. Regras para análise de sementes. Brasília, DF: SNAD/ DNDV/CLAV, 1992. 365 p.

CARVALHO, N. M.; NAKAGAWA, J.Sementes: ciência, tecnologia e produção. 4. ed. Jaboticabal: Funep, 2000. 588 p.

DAYAL, B. R.; KAVERIAPPA, K. M. Effect of desiccation and temperature on germination and vigour of the seeds of Hopea parviflora Beddome and $H$. ponga (Dennst.) Mabb. Seed Science \& Technology, Zurich, v. 28, n. 2, p. 497-506, 2000.

DOUSSEAU, S.; ALVARENGA, A. A. DE.; ARANTES, L. DE O.; OLIVEIRA, D. M. DE; NERY, F. C. Germinação de sementes de Tanchagem (Plantago tomentosa Lam.): influência da temperatura, luz e substrato. Ciência e Agrotecnologia, v.32, n.2, p. 438-443, mar./abr. 2008.

FARRANT, J. M.; PAMMENTER, N. W.; BERJAK, P.; WALTERS, C. Subcellular organization and metabolic activity during the development of seeds that attain different levels of desiccation tolerance. Seed Science Research, Wallingford, v. 7, n. 2, p. 135-144, June 1997.

FERREIRA, A. G.; BORGHETTI, F. Germinação: do básico ao aplicado. Porto Alegre: Artmed, 2004. 323 p.

FERREIRA, D. F. Análises estatísticas por meio do Sisvar para Windows versão 4.0. In: REUNIÃO ANUAL DA REGIÃO BRASILEIRA DA SOCIEDADE INTERNACIONAL DE BIOMETRIA, 45., 2000, São Carlos. Anais... São Carlos: UFSCar, 2000. p. 255-258.

HONG, T. D.; ELLIS, R. H. A comparison of maturation drying, germination, and desiccation tolerance between developing seeds of Acer pseudoplatanus L. and Acer platanoides L. New Phytologist, Cambridge, v. 116, n. 4, p. 589-596, Dec. 1990.

KUNDU, M.; KACHARI, J. Desiccation sensitivity and recalcitrant behavior of seeds of Aquilaria agallocha Roxb. Seed Science \& Technology, Zurich, v. 28, n. 3, p. 755-760, 2003.

LIMA JÚNIOR, E. C. Germinação, armazenamento de sementes e fisioanatomia de plantas jovens de Cupania vernalis Camb. 2004. 115 p. Dissertação (Mestrado em Agronomia Fisiologia Vegetal) - Universidade Federal de Lavras, Lavras, 2004. 
MAGUIRE, J. D. Speed of germination aid in selection and evaluation for seedling emergence and vigor. Crop Science, Madison, v. 2, n. 2, p. 176-177, Mar./Apr. 1962.

MALUF, A. M.; MARTINS, P. S. Germinação de sementes de Amaranthus hybridus L. e Amaranthus viridis L. Revista Brasileira de Biologia, Rio de Janeiro, v. 51, n. 3, p. 417-425, ago. 1991.

NERY, F. C. Aspectos da germinação, armazenamento de sementes, crescimento inicial e anatomia de plantas jovens de Calophyllum brasiliense Cambess. 2006. 173 p. Dissertação (Mestrado) - Universidade Federal de Lavras, Lavras, 2006.

NERY, F. C.; ALVARENGA, A. A. DE; JUSTO, C. F.; DOUSSEAU, S.; VIEIRA, C. V. Efeito da temperatura e do tegumento na germinação de sementes de Calophyllum brasiliense. Ciência e Agrotecnologia, v.31, n.6, p. 18721877, nov./dez. 2007

SCATENA, V. L.; LEMOS, F. J. P.; LIMA, A. A. A. Morfologia do desenvolvimento pós-seminal de Syngonanthus elegans e S. niveus (Eriocaulaceae). Acta Botânica Brasílica, São Carlos, v. 10, n. 1, p. 85-91, jan./abr. 1996.

SCOTT, A. J.; KNOTT, M. Cluster analysis method for grouping means in the analysis of variance. Biometrics, Washington, v. 30, n. 3, p. 507-512, 1974.
THOMPSON, P. A. Characterization of the germination response to temperature of species and ecotypes. Nature, London, v. 225, p. 827-831, 1970.

TIGABU, M.; ODEM, P. C. Effect of scarification, giberellic acid and temperature on seed germination of two multipurpose Albizia species from Ethiopia. Seed Science and Technology, Zurich, v. 29, n. 1, p. 11-20, 2001.

VARGHESE, B.; NAITHANI, R.; DULLOO, M. E.; NAITHANI, S. C. Seed storage behaviour in Madhuca indica J. F. Gmel. Seed Science \& Technology, Zurich, v. 30, n. 1, p. 107-117, 2002.

VIEIRA, C. V.Sensibilidade à dessecação, armazenamento, germinação e morfologia de sementes de Cupania vernalis Camb. 2005. 65 p. Dissertação (Mestrado em Fisiologia Vegetal) - Universidade Federal de Lavras, Lavras, 2005.

VILLALOBOS, A. E.; PELÁEZ, D. V. Influences of temperature and water stress on germination and establishment of Prosopis caldenia Burk. Journal of Arid Environment, London, v. 49, n. 2, p. 321-328, Oct. 2001.

ZAR, J. H. Biostatistical analysis. 4. ed. New York: Prentice Hall, 1999. 929 p. 